ORIGINAL ARTICLE

\title{
National Health Insurance Scheme: An Assessment of Service Quality and Clients' Dissatisfaction
}

\author{
Ajeigbe Abiodun Kofoworola ${ }^{*}$, Ayinbuomwan Ekiye ${ }^{2}$, Adesina Olufunlola \\ Motunrayo $^{3}$, Adedeji Tewogbade Adeoye ${ }^{1}$, Makinde Ronke Adunni ${ }^{1}$
}

\section{OPEN ACCESS}

Citation: Ajeigbe Abiodun Kofoworola, Ayinbuomwan Ekiye, Adesina Olufunlola Motunrayo, Adedeji Tewogbade Adeoye, Makinde Ronke Adunni. National Health Insurance Scheme: An Assessment of Service Quality and Clients' Dissatisfaction. Ethiop J Health Sci.2020;30(5):795. doi:http:// dx.doi.org/ 10.4314/ejhs.v30i5.20

Received: February 15, 2020

Accepted: April 15, 2020

Published: September 1, 2020

Copyright: (C) 2020 Ajeigbe A.K., et al. This is an open access article distributed under the terms of the Creative Commons Attribution License, which permits unrestricted use, distribution, and reproduction in any medium, provided the original author and source are credited.

Funding: Nil

Competing Interests: The authors declare that this manuscript was approved by all authors in its form and that no competing interest exists.

Affiliation and Correspondence:

${ }^{1}$ Department of Chemical Pathology,

Obafemi Awolowo University (OAU), Ile-Ife

${ }^{2}$ Department of Chemical Pathology, University of Benin (UNIBEN), Benin-City

${ }^{3}$ Department of Oral and Maxillofacial Surgery/Oral Pathology, OAU, Ile-Ife

*Email: abiodunalaje1@gmail.com

\section{ABSTRACT}

BACKGROUND: Health Insurance Scheme (NHIS), a medical package to start with a fraction of Nigerians at its inception, with the expectation of optimal services to all in the long The Nigerian government established National run. However, there are complaints and dissatisfaction of enrolees of the scheme. This study determined factors responsible for enrolees' dissatisfaction of services in a general hospital within the federal capital territory, Abuja.

METHOD: Three hundred enrolees of National Health Insurance Scheme at the Kubwa general hospital were enrolled. Semi structured questionnaires were used to obtain information on socio-demography, education, enrolee status, perception of the scheme and factors responsible for enrolees' dissatisfaction. SPSS version 22 was used to analyse Data using percentage.

RESULTS: Majority (66.3\%) of the respondents were between 35 and 54 years while $28.5 \%$ were below 35years and $11.8 \%$ (32) were above $54 y e a r s$ with the male to female ratio was 1.03:1. Sixty percent (179) of the respondents had a minimum of tertiary education with just $1.8 \%$ having primary education. Most (69.9\% and 79.6\%) respondents were principal enrolees and public servants respectively. Seventy percent of the respondents have been enrolled in NHIS for more than 3years and had a good perspective of the scheme. However, $30 \%$ of the respondents were not satisfied with NHIS services with $8.6 \%$ and $15.4 \%$ describing the services as substandard and below expectations respectively. Half (50.7\%) of the respondents would consider an alternative to NHIS suggesting their dissatisfaction. The major factors responsible for dissatisfaction were billing system, long waiting hours and staff attitude accounting for $46.9 \%, 59.4 \%$ and $7.8 \%$ respectively. CONCLUSION: This study revealed that the level of clients' dissatisfaction with NHIS services is high despite their acceptance of the scheme with the major areas of concern being the billing system, waiting time and staff attitude. Therefore, it is necessary for the providers to look more into these areas as targets for service delivery improvement.

KEYWORDS: National Health Insurance Scheme, Dissatisfaction, Service delivery. 


\section{INTRODUCTION}

Provision of free health for all is a major challenge in Nigeria. Although government officials and students have long benefitted from subsidized healthcare services, the general population still pay for these services. Since the introduction of National Health Insurance Scheme (NHIS) in 2005 (1) based on contributory finance, it is believed that the pooled funding would cater for infrastructure, manpower and equipment necessary for clients' health needs (2). However, the clients' experience of NHIS services over the years is unsatisfactory as the majority of enrolees are displeased and not satisfied with services rendered. Therefore, this has resulted in accessing alternative health facilities where outright out of pocket (OOP) payment would be made. Thus, it is important to identify those areas causing clients' dissatisfaction as targets for service improvement. The need for NHIS in Nigeria was first recognized in $1962(1,3)$, approved in 1997, passed into law in 1999 and officially launched on $6^{\text {th }}$ June 2005 . The objective of NHIS is to provide comprehensive healthcare at affordable costs with limited percentage of citizens covered (government and private employees, vulnerable groups). Based on this, it is important to measure health system effectiveness as it aligns to improvements in access to quality healthcare and client satisfaction (4). Patient satisfaction is defined as patient's judgement on the quality and goodness of care. It is the best possible health outcome given the available resources consistent with patient values and preferences. Patients, being the healthcare services consumers, have expectations and needs warranting satisfaction (5). This determines the quality of care by healthcare providers which is a professional responsibility. NHIS enrollees' dissatisfaction in Nigeria is yet to be fully explored necessitating the need to evaluate causes and factors associated with enrolees' dissatisfaction of healthcare services using selected indices of satisfaction in the hospital. This is with the view of improving service delivery within the hospital settings

Previous studies evaluating the NHIS performances reported that there were complaints where providers denied enrolees their full entitlements and some providers charged additional fees (6-8). Another study described enrolees' experiences as unsatisfactory with inadequate drugs, poor registration services and referral system as well as delayed services as reasons (7) for their dissatisfaction. It has been found that waiting time and attitude of healthcare staff were predictors of users' satisfaction of NHIS (9). Similarly, in the evaluation of NHIS in 2006, the major areas of clients' dissatisfaction were poor attitude and behaviour of service providers (10). Another study in Ibadan reported that $54.7 \%$ of their respondents were dissatisfied with the process of enrolment, $50.5 \%$ with range of services, $62.1 \%$ with the co-payment plan, $55.4 \%$ with change of provider and an overall dissatisfaction of $16.4 \%$ (11). The report from Jos was that $26 \%$ of their respondents were dissatisfied, necessitating an immediate attention from providers and Health Maintenance Organizations (HMOs), with sources of dissatisfaction being poor registration services, poor referral system, delay of services and unavailable services (12). Prolonged waiting time was a major cause of dissatisfaction with observations of inefficiency and ineffectiveness in some areas of operations (7,11). A similar study in Enugu by Iloh reported that bureaucracy with prolonged waiting time was a major concern (13). Patient satisfaction being a dominant concern mentioned with strategic health services decisions (WHO) requires evaluation. Surveys on satisfaction have been widely used to address access and performance (14-16) which has helped government agencies to measure performance (17). It has been suggested that there is a need for continuous monitoring of clients' satisfaction and dissatisfaction (18) through research to impact future implementation strategies which can be imported to the healthcare system. We therefore assessed the factors responsible for NHIS enrolees' dissatisfaction in a general hospital setting at the Federal Capital Territory which is supposed to be a pace setter of quality service delivery for other centres to emulate within the country.

\section{METHODS}

This is a descriptive cross-sectional study conducted at the NHIS clinic of Kubwa General Hospital (KGH), Abuja, between November 2016 and March 2017. The hospital is one of the secondary health facilities within the Federal 
capital development authority having a 200 bed capacity. It is a major referral centre to neighbouring general hospitals and primary health centres. The population for this study were all enrolees of NHIS between 18 and 60 years attending $\mathrm{KGH}$ as the primary healthcare provider. The estimated enrolees were 5000 within six months and above. Excluded from the study were those who joined the scheme less than 6 months and did not visit clinics during the period of study. The calculated sample size was 295.6 using Leslie-Kish formula: $n=Z \alpha 2 p q / d 2$ for descriptive study using $26 \%$ proportion of enrolees satisfaction of NHIS from a previous study by Onyedibe (7). A total of 300 enrolees were sampled using simple random sampling. Data was collected from consenting enrolees using questionnaires. Data was analysed with the Statistical Package for Social Sciences (SPSS) version 22. Likert scales were used to assess the degree of agreement and disagreement with statements in the questionnaire. Chi-square was used to assess the relationship between the variables of dissatisfaction and sociodemographic parameters, and a $\mathrm{P}<0.05$ was taken as statistically significant. Results were presented using tables. A pre-test was done to assess the component of questionnaire for data collection and respondents' reactions. Ethical clearance was obtained from the Ethical and Research Committee of KGH, Abuja, and written informed consent was obtained from respondents.

\section{RESULTS}

Three hundred subjects were given selfadministered questionnaires to fill with $93 \%$ response rate. Enrolees between 25 and 54 years of age formed the majority (90.5\%) of our respondents with $4.3 \%$ below 24 years and $11.8 \%$ above 54 years reflecting that youths and middle aged constitute the bulk of the labour force in Nigeria. Males (50.9\%) and females of the workforce (Table 1).
Table 1: Distribution of age and sex of respondents $(\mathrm{n}=279)$.

\begin{tabular}{|ll}
\hline Variable & Frequency (\%) \\
\hline AGE (years) & \\
$\mathbf{1 5 - 2 4}$ & $12(4.3)$ \\
$\mathbf{2 5 - 3 4}$ & $50(17.9)$ \\
$\mathbf{3 5 - 4 4}$ & $102(36.6)$ \\
$\mathbf{4 5 - 5 4}$ & $83(29.7)$ \\
$\mathbf{5 5 - 6 4}$ & $32(11.5)$ \\
Sex & \\
Male & $142(50.9)$ \\
Female & $137(49.1)$ \\
\hline
\end{tabular}

About $70 \%$ and $80 \%$ of the respondents were principal enrolees of NHIS from government establishments respectively suggesting a good health-seeking behaviour and support from government. The majority (78.1\%) of the respondents were married in a monogamous $95 \%$ family setting. Less than $50 \%$ of the respondents had been enrolled for over 5 years with $58.2 \%$ enrolled for 5 years (Tables 2 and 3 ).

Table 2: Educational and NhIS enrollee status $(n=279)$.

\begin{tabular}{ll}
\hline Variable & Frequency (\%) \\
\hline Educational Level & \\
Primary & $5(1.8)$ \\
Secondary & $95(34.1)$ \\
Tertiary & $179\left(64.2^{*}\right)$ \\
NHIS Status & \\
Principal & $195(69.9)$ \\
Dependent & $84(30.1)$ \\
Enrolment & \\
period(year) & \\
$<1$ & $18(6.5)$ \\
$1-3$ & $26(9.3)$ \\
$3-5$ & $110(39.4)$ \\
$>5$ & $125(44.8)$ \\
Employment Status & \\
Government & $222\left(79.6^{*}\right)$ \\
Private & $13(4.6)$ \\
Self Employed & $22(7.9)$ \\
Dependent & $22(7.9)$ \\
\hline
\end{tabular}


Table 3: Marital and family status of respondents $(\mathrm{n}=279)$.

\begin{tabular}{ll}
\hline Variable & Frequency (\%) \\
\hline Marital Status & \\
$\quad$ Single & $61(21.9)$ \\
$\quad$ Married & $218(78.1)$ \\
Family setup & \\
Monogamous & $265(95)$ \\
$\quad$ Polygamous & $14(5)$ \\
Family size & \\
$<6$ & $204(73.1)$ \\
$>6$ & $75(26.9)$ \\
\hline
\end{tabular}

Most (74.7\%) respondents perceived services of NHIS as quality although (49.1\%) were evenly distributed suggesting gender balance $22.9 \%$ were not satisfied with the services while $7.9 \%$ of the respondents were undecided. More than half $(50.7 \%)$ of the respondents would prefer an alternative to NHIS while the majority wanted $(88.4 \%)$ NHIS sustained and improved (Table 4).

Table 4: Quality and preference for NHIS services $(n=279)$.

\begin{tabular}{ll}
\hline Variable & Frequency (\%) \\
\hline Quality of Services & \\
Below expectation & $43(15.4)$ \\
Substandard & $24(8.6)$ \\
Standard & $177(63.4)$ \\
Quality & $35(12.5)$ \\
Responses to quality & \\
service & \\
Undecided & $14(5.1)$ \\
Disagree & $9(3.2)$ \\
Agree & $207(74.7)$ \\
Strongly agree & $47(17)$ \\
Non-response & $2(0.7)$ \\
Preference for NHIS & \\
Never & $0(0)$ \\
Maybe & $140(50.7)$ \\
Definitely Yes & $136(49.3)$ \\
Non-response & 3 \\
\hline
\end{tabular}

The reasons for dissatisfaction were billing system, waiting time and staff attitude accounting for $46.9,59.4$ and $7.8 \%$ respectively. Two hundred and six of respondents spent most time waiting for consultation followed by laboratory tests $(9.8 \%)$, nurses station $(6.2 \%)$ and medical records (5.8\%). Most (48.5\%) were not pleased with the attitude of staff in records station followed by those in pharmacy $(26.2 \%)$, laboratory (13.6\%), nursing (6.8\%) and radiology $(4.9 \%)$ (Table 5).

Table 5: Areas of dissatisfaction $(\mathrm{n}=279)$.

\begin{tabular}{ll}
\hline Variable & Frequency (\%) \\
\hline BILLING SYSTEM & \\
Undecided & $2(0.7)$ \\
Uncomfortable & $130(46.9)$ \\
Very comfortable & $145(52.4)$ \\
Non-response & 2 \\
Waiting Time & \\
$<1$ & $109(39.1)$ \\
$1-2$ & $10(3.6)$ \\
$2-3$ & $22(7.9)$ \\
$3-4$ & $138(49.4)$ \\
Staff Attitude & \\
Undecided & $2(0.7)$ \\
Dissatisfied & $21(7.8)$ \\
Satisfied & $205(76.5)$ \\
Very Satisfied & $40(14.9)$ \\
Non-response & 11 \\
\hline
\end{tabular}

Most respondents (48.9\%) would like to see an improvement on the waiting time at the pharmacy services $(32.2 \%)$, laboratory $(14.8 \%)$ and billing $(4.2 \%)$ (Table 6). This improvement would increase the satisfaction of clients as the services would be perceived robust. 
Table 6: Sources of dissatisfaction $(n=279)$.

\begin{tabular}{|c|c|}
\hline Variable & Frequency (\%) \\
\hline \multicolumn{2}{|l|}{ Waiting time } \\
\hline Medical records & $16(5.8)$ \\
\hline Nurses station & $17(6.2)$ \\
\hline Consulting room & $216(78.3)$ \\
\hline Laboratory & $27(9.8)$ \\
\hline Non-response & 3 \\
\hline \multicolumn{2}{|l|}{ Professional sources } \\
\hline Records staff & $50(48.5)$ \\
\hline Nursing staff & $7(6.8)$ \\
\hline Lab staff & $14(13.6)$ \\
\hline Pharmacy staff & $27(26.2)$ \\
\hline Radiology staff & $5(4.9)$ \\
\hline Medical staff & 0 \\
\hline Non-response & 176 \\
\hline \multicolumn{2}{|l|}{$\begin{array}{l}\text { Prof. sources of } \\
\text { satisfaction }\end{array}$} \\
\hline All professionals & $17(7.9)$ \\
\hline Lab staff & $2(0.9)$ \\
\hline Records staff & 26 \\
\hline Medical Staff & 170 \\
\hline Non-response & 64 \\
\hline Areas & \\
\hline \multicolumn{2}{|l|}{ Improvement } \\
\hline Waiting Time & $129(48.9)$ \\
\hline Pharmacy services & $85(32.2)$ \\
\hline Lab. Services & $39(14.8)$ \\
\hline Billing & $11(4.1)$ \\
\hline Non-response & 15 \\
\hline
\end{tabular}

\section{DISCUSSION}

The age distribution of the respondents in this study clearly reflects the coverage of all age groups by NHIS in line with its objectives. It also shows that NHIS is mostly accessed by those in the working age of 25-60 years who are primary enrolees. It is also obvious that the workforce in Nigeria is between 25 and 54 years with a peak of 35-44 years. There is also a wide coverage to capture the dependents of primary enrolees. Males and females in this study had similar health seeking attitude which was good. This may be due to the impact of the educational level of the respondents in which the majority were found to have a minimum of tertiary education. This in turn might be a reflection of a high literacy level of city dwellers and because NHIS captures skilled workers in government establishments. This supports earlier findings by Kayode et al (11). In this study, it was observed that the principal enrolees were more than the dependents probably due to the fact that they were primary targets and government workers constituting more than two-third of the enrolees compared to other workers. This is similar to reports from Jos by Shaffiu (12). Although NHIS kicked off in 2015 with the formal government sector which is just less than $25 \%$ of the population with the projections of expanding the scheme to include the informal sector, it is surprising that NHIS is still struggling to provide healthcare services to a few Nigerians more than 10 years after its inception. Neglected citizens include artisans, traders, transporters, farmers and market women who are yet to benefit from NHIS. Akeem in his study among artisans in Lagos in 2011 determined the reactions of these group to NHIS and observed that these citizens felt neglected and marginalized (19). There have been reports that these groups can access NHIS services, but the package is not robust and the protocol is often ambiguous leading to frustration of individuals. This study found that NHIS was attended by married enrolees probably because of access by dependent family members. More married people had monogamous family settings with a larger family size which could help NHIS make forecast and plan for the future services. The respondents in this study have a larger percentage of enrolees of more than 3 years suggesting that they may have a better and clearer perspective of NHIS services and give better information. Kayode et al reported that clients with longer duration of enrolment were more satisfied with waiting time and staff attitude (11).

The majority of the respondents agreed to NHIS provision of quality services but some of the enrolees are still unsatisfied. Shaffiu in 2011 reported similar satisfaction $(61.5 \%)$ and dissatisfaction (26\%) rates supported by Iloh who reported overall satisfaction rate of $66.5 \%$ among his study participants in South-eastern Nigeria in 2016 (12-13). Similarly, Onyedibe et al reported $61.5 \%$ satisfaction with NHIS services and $26 \%$ dissatisfaction in the participants of a study in 
2012 (7). However, the report from Ibadan by kayode et al revealed that fluctuating levels of satisfaction with NHIS services were dependent on components being examined (11). This study confirms earlier reports of higher rates of NHIS services satisfaction than dissatisfaction. Although, many clients appear to be satisfied with services rendered by NHIS, there is a need for necessary action to improve the clients' dissatisfaction. This is because the level of dissatisfaction of NHIS enrolees is still high and may even be higher if different separate components of NHIS services are examined independently. Thus, suggesting a need for indepth NHIS services evaluation and assessment of its different units or sections as areas for targeted improvements. It was also observed that most respondents were willing to continue patronage of NHIS in the presence of alternatives suggesting good service delivery. Also, participants found NHIS billing comfortable, and it eased the burden of OOP payment which could be difficult for salary earners. On the other hand, many participants were uncomfortable with the billing system due to frequent out-of-stock drugs in the pharmacy necessitating OOP purchase outside the service centres. This unsatisfied group felt cheated as they had fully paid their contributory fund. This suggests that these individuals could not get value for their money.

Most time was spent at the consulting room waiting to see the doctor. This is due to the fact that few doctors are now available in Nigeria. It was reported that the ratio of doctor-to-patient is $1: 53,333$ in Nigeria buttressing the shortage of this highly skilled professionals due to brain drain as a result of poor work conditions, lack of welfare, lack of motivation and poor remuneration. More so, NHIS clinics utilize medical officers within the domiciled hospitals who have other clinical roles. Despite the prolonged time waiting to see the doctor, it was observed that most respondents were mostly pleased with doctors' attitude followed by the attitudes of record staff and the laboratory staff the least. This supports earlier reports by Iloh and Ofili $(13,20)$. On the other hand, half of the respondents were not pleased with the attitude of staff in records, pharmacy, laboratory, nursing and radiology units. This finding may be two way viz the staff and the client factors. This may be due to the fact that the records station is the first point of contact for most enrolees, who are usually in a haste. Often times, record staff might be unwilling to follow protocol in case file retrieval for most patients. The staff in this section also handle lots of patient information which include data on registration, retrieval/ filing of new clients folder, archive of important documents, generation of approval codes for investigations and referrals as well as stamping of prescription forms. They are also saddled with responsibility of authorization of referral forms, liaisons between enrolee and HMOs among other functions. In addition, there are usually fewer personnel to man this station and handle numerous administrative roles, eventually overwhelming the staff and thus, their disposition to the enrolees may be perceived as poor. Thereby, resulting in frequent friction between enrolees and officers. The medical records, being an integral component of NHIS, require necessary attention and periodic staff performance evaluation and training to improve this area of service delivery. Many of the respondents in this study desire an improvement of NHIS services with most emphasis on the waiting time at the clinics, pharmacy and the laboratory. It is therefore important for stakeholders to look deeply into these areas and make proper adjustments.

The number of absent responses to questions requiring enrolees' comment on their satisfaction with different professional groups was low compared to the responses to other questions. This might be due to a general perception in our environment not supporting negative comments on staff especially within a public setup. It is believed that such comments could cause dismissal of such employee. Thus, clients will rather keep mute than give a contrary opinion regarding wrong attitude of public servants in discharging their duties. This may also suggest the basis of gross indiscipline and impunity in government establishments. However, if we desire an improvement in our public services, there must be healthy criticisms from users of such services. Therefore, these may be areas of great importance to future research.

This study has some limitations. One is nonrecruitment of NHIS staff members who are also enrolees or potential enrolees of the scheme. This 
is because of the perceived possibility of them being biased in answering the questions on satisfaction of services they rendered. Another limitation is the study being single-centred, as a multi-centred study may give a broader picture of clients' satisfaction.

In conclusion, in spite of the above stated limitations, it could be conclusively inferred from this study that evaluation of healthcare service delivery should be a continuous exercise to ensure quality service delivery or clients' satisfaction. NHIS should expand their services to capture the non-skilled workforce, and remove the bottlenecks associated with registration. It is hoped that different researchers would find NHIS service delivery as an area of further research.

\section{REFERENCES}

1. Katibi I.A, Akande A.A. Akande T.M. Awareness and attitude of medical practitioners in Ilorin towards National Health Insurance Scheme. Sahel Medical Practice. 2003;6:1.

2. FMH. Operational Guideline of National Health Insurance Scheme. FMH Abuja. 2005.

3. Akande T.M, Bello O. National Health Insurance Scheme in Nig. Medilor $J$. 2002;7:1.

4. Kruk M.E, Freedman L.P. Assessing health system performance in developing countries: A review of the literature. Health Policy. 2008;85:263-76.

5. Liyasu Z, Abubakar I.S, Abubakar S, Lawan U.M, Gajida A.U. Patients' satisfaction with services obtained from Aminu Kano Teaching Hospital, Kano, Northern Nigeria. Nigeria Journalof Clinical Practice. 2010;13:371-8.

6. Lagomarsino $G$, Garabrant A, Adyas A, Muga R, Otoo N. Moving towards universal health coverage: health insurance reforms in nine developing countries in Africa and Asia. Lancet.2012; doi: $\quad 10.1016 / \mathrm{S} 0140$ 6736(12)61147-7.

7. Onyedibe K.I, Goyit M.G, Nnadi N.E (2012). An evaluation of the national health insurance scheme (NHIS) in Jos, a NorthCentral Nigerian city. Global Advanced
ResearchJournal of Microbiology. 2012;1 Suppl 1: 005-12.

8. Nabbuye-Sekandi J, Makumbi FE, Kasangaki A, Kizza IB, Tugumisirize J, Nshimye E, et al. Patient satisfaction with services in outpatient clinics at Mulago hospital, Uganda. Internationla Journal of Quality Health Care. 2011;23 Suppl 5:51623.

9. Wouters E, Heunis C, van Rensburg D, Meulemans H. Patient satisfaction with antiretroviral services at primary health-care facilities in the Free State, South Africa--a two-year study using four waves of crosssectional data. BMC Health Serv Res. 2008;8:210.

10. National Health Insurance Scheme. National health insurance scheme annual report. National Health Insurance Scheme Abuja, Nigeria. 2006.

11. Kayode O. Osungbade, Taiwo A. Obembe and AbidemiOludoyi. Users' Satisfaction with Services Provided Under National Health Insurance Scheme in South Western Nigeria.International Journal of Tropical Disease and Health. 2014;4 Suppl 5:595607.

12. Shaffiu M, Mohammad N Sambo, Hengjin Dong. Understanding client satisfaction with a health insurance scheme in Nigeria: factors and enrollees experiences. Health Research Policy and System. 2011;9 Suppl 20:1-8.

13. Iloh G.U.P, J.N Ofoedu, P.U Njoku, F.U Odu, C.V Ifedigbo, K.D Iwuamanam. Evaluation of patients' satisfaction with quality of care provided at the National Health Insurance Scheme clinic of a tertiary hospital in South- Eastern Nigeria. Nigerian Journal of Clinical Practice. 2012;15 Suppl 4:469-474.

14. Andaleeb S.S. Service quality perceptions and patient satisfaction: a study of hospitals in a developing country. Social Science and Medicine. 2001;52:1359-137

15. Oladapo T.O, Iyaniwura A.C, Sule-Odu O.A. Quality of antenatal services at the primary care level in Southwest Nigeria. African Journal of Reproductive Health. 2008;12:7192. 
16. Myburgh NG, Solanki GC, Smith MJ, Lalloo R (2005).Patient satisfaction with health care providers in South Africa: the influences of race and socioeconomic status. International Journal of Quality Health Care. 2005;17 Suppl 6:473-7.

17. Langseth P, Langan P, Talierco R: Service delivery survey (SDS): a management tool. The Economic Development Institute of the World Bank. 1995.

18. Huber G. Mutual health insurance (MHO). five years experiences in West Africa: concerns and controversies. Deutsche
Gesellschaft fur TechnischeZusammenarbeit (GTZ). 2002.

19. 19. Akeem A.A, Adedoyin S, Olasunmbo O. Artisans' reactions to national health insurance scheme in Lagos State, Nigeria.The Journal of Global Health Care Systems. 2014; $14: 1$.

20. 20. Ofili A.N, Ofovwe C.E. Patients'assessment of efficiency of services at a teaching hospital in a developing country. Annals of African Journal. 2005;4:15. 